Contributions to Game Theory and Management, XIV, 59-71

\title{
New Characteristic Function for Two Stage Games with Spanning Tree
}

\author{
Min Cheng ${ }^{1}$ and $\mathrm{Yin} \mathbf{L i}^{1,2}$ \\ 1 St. Petersburg State University, \\ 7/9 Universitetskaya nab., Saint Petersburg, 199034, Russia \\ 2 School of Economics and Management, Yanan University, Yan'an, 716000, China \\ E-mail: cm014033@163.com, liyinrus@outlook.com
}

\begin{abstract}
Two-stage $n$-player games with spanning tree are considered. The cooperative behaviour of players is defined. After the first stage, a specified player leaves the game with a probability that depends on the actions of all players in the first stage. A new approach to the construction of the characteristic function is proposed. In the game, all players are connected with the source directly or indirectly. Assume that the players in coalition $N \backslash S$ have already connected to the source when the players in coalition $S \subset N$ wish to connect to the source. The players in coalition $S$ could connect to the source with the help of the players in coalition $N \backslash S$. A new characteristic function is defined in the game, and the Shapley value is constructed. Several results based on the new characteristic function in the two-stage stochastic game are given.
\end{abstract}

Keywords: dynamic game, minimum cost spanning tree, Shapley value

\section{Introduction}

In the minimum cost spanning tree game, it is considered that a group of players need to connect the source to get some service or benefit and share the total cost between them. In (Bird, 1976), it is the first time to propose a groundbreaking method to solve this problem, namely the Bird rule, and it is a cost allocation rule in the game with spanning tree. After this, different kinds of solutions in the game with spanning tree have been proposed. Such as the core and nucleolus (Granot and Huberman, 1984), the Folk solution (Feltkamp et al., 1994), the Kar rule (Kar, 2002), and the fair rule (Bergantiños et al, 2007a).

Especially in (Bergantiños et al., 2007b), the author considered that the players in coalition $S \subset N$ might indirectly connect to the source with the help of the players in the coalition $N \backslash S$, while the players in the coalition $N \backslash S$ already connected with the source. The game described before is named as the "optimistic" minimum cost spanning tree game. ${ }^{1}$ In this paper, it is considered to construct the Shapley value in the "optimistic" game.

In ( $\mathrm{Li}, 2016)$, a two-stage spanning tree game with shock is considered, and assumed that after the first stage in the game, a particular player would leave the game with a probability that depends on the situation in the first stage. In this research the characteristic functions for coalitions are defined by the Bird method(Bird, 1976). The dynamic Shapley value is considered as a solution in the game. In (Yin, 2017), a two-stage spanning tree game with perishable products is studied. In the game, all players need to share the total cost of edges on the minimum cost spanning

\footnotetext{
${ }^{1}$ The corresponding "pessimistic" game means that the players in coalition $S$ are connected to the source without the help of others.
}

https://doi.org/10.21638/11701/spbu31.2021.05 
tree and the loss caused by the perishable products. The research concluded that the dynamic Shapley value is time inconsistent in the two-stage game with perishable products. Also the two-stage game with forest is studied(Yin, 2017). In this research, there are $n$ players and $m \geq 2$ sources in the game. It is proved that using the imputation distribution procedure (IDP)(Petrosyan, 1979), the dynamic Shapley value constructed in the game will be time consistent if there is at least one player who wants to connect to all sources. Furthermore, in (Yin, 2020), the twostage stochastic game with spanning tree is present. In this research, a transition matrix determined by the players is introduced.

In the paper, an "optimistic" two-stage game with spanning tree is considered. The players in the coalition $S \subset N$ no longer need to connect with the source directly. If necessary, the coalition $S$ can indirectly connect with the source by the players who are not in the coalition $S$. At the beginning of the game, an initial cost matrix is given. In each stage game, arbitrary two players can take actions to redefine the cost of the edge between them. For any two players, if they did not take action together to change the cost of the edge, then the cost of the edge between them will remain fixed as the cost of the edge from the initial cost matrix at the beginning of the game. All players choose their actions simultaneously at the first stage. As shown in Fig.1, after the first stage, a particular player $m$ may leave the game with probability $p$. The probability is defined by actions of all players in the first stage.

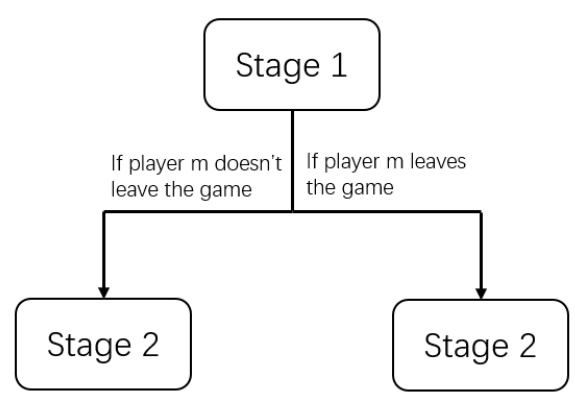

Fig. 1. Process of the game

\section{The model}

\subsection{Basic definitions}

Let $N=\{1,2, \ldots, n\}$ be a finite set of players. The source is denoted by $\{0\}$, and $N^{\prime}=N \cup\{0\}$. A graph over $N^{\prime}$ is denoted by $G=\left(N^{\prime}, E\right)$. $E$ is the set of all edges. A pair $(i, j)$ is called an edge in $G\left(N^{\prime}, E\right)$, if $(i, j) \in E, \forall i, j \in N$. A coalition $S$ is the subset of $N$, where $S \subseteq N$ and $S^{\prime}=S \cup\{0\}$.

Definition 1. A path in a graph is a finite or infinite sequence of edges that links a set of distinct vertices. If there is a path from vertex $i$ to $j$, then $i$ and $j$ are said to be connected. The graph is called a connected graph if any two vertices in a graph are connected. 
Definition 2. $G\left(N^{\prime}, E\right)$ (or $G\left(S^{\prime}, E\right)$ ) is defined as the connected graph over $N^{\prime}$ (or $S^{\prime}$ ). $\mathcal{G}_{N^{\prime}}$ (or $\mathcal{G}_{S^{\prime}}$ ) is a set of connected graphs with vertices in $N^{\prime}$ (or $S^{\prime}$ ), where $S \varsubsetneqq N$.

Example 1. Consider an example. $N=\{1,2,3,4\}, S=\{1,2,3\} \subset N, N^{\prime}=N \cup\{0\}$, $S^{\prime}=S \cup\{0\},\{0\}$ is the source. As shown in Fig.2, which represents two different connected graphs $\dot{G}_{N^{\prime}}$ and $\ddot{G}_{N^{\prime}}$ over $N^{\prime}$, and Fig.3 shows two connected graphs $\dot{G}_{S^{\prime}}$ and $\ddot{G}_{S^{\prime}}$ over $S^{\prime}$.
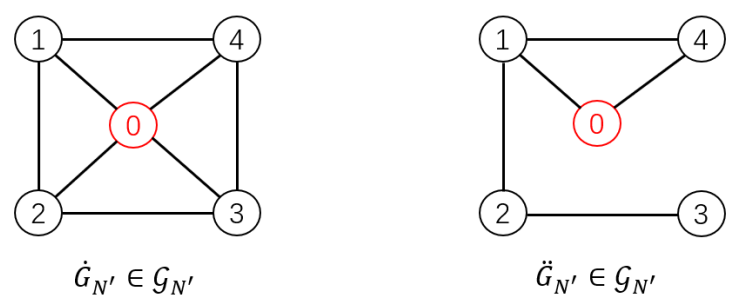

Fig. 2. Connected graphs over $N^{\prime}=\{0,1,2,3,4\}$
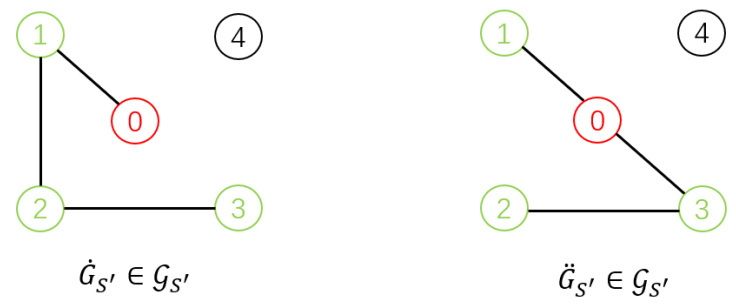

Fig. 3. Connected graphs over $S^{\prime}=\{0,1,2,3\}$

$C=\left(c_{i j}\right)_{(n+1) \times(n+1)}$ is a cost matrix, in which all elements represent the costs of edges between vertexes on the graph $G\left(N^{\prime}, E\right) \cdot c_{i j}=c_{j i}>0$ is the cost of edge $(i, j), \forall i, j \in N^{\prime}$.

The cost matrix associated with $G\left(N^{\prime}, E\right)$ (or $G\left(S^{\prime}, E\right)$ ) is denoted by $C$ (or $\left.C^{S}\right)$.

Definition 3. In $G\left(N^{\prime}, E\right)$, an initial cost matrix $C^{0}$ is defined as

$$
C^{0}=\left(c_{i j}^{0}\right)_{(n+1) \times(n+1)}, c_{i j}^{0}=c_{j i}^{0}>0, \forall i \neq j \in N^{\prime}
$$

Consider an example about the initial cost matrix.

Example 2. As shown in Fig.4, $N=\{1,2,3,4,5,6\}, N^{\prime}=N \cup\{0\}$. 


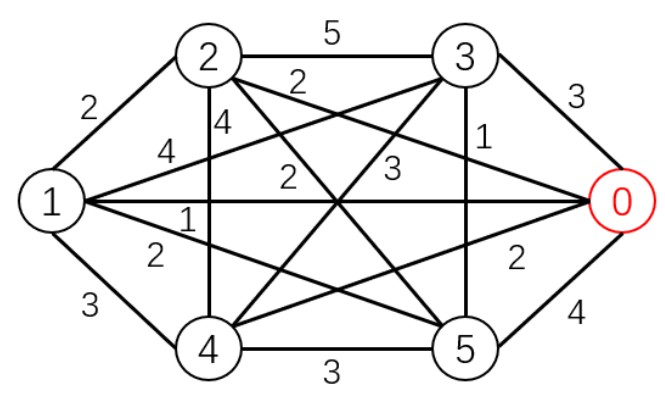

Fig. 4. Initial cost matrix $C^{0}$

The initial cost matrix associated with the graph $G\left(N^{\prime}, E\right)$ is

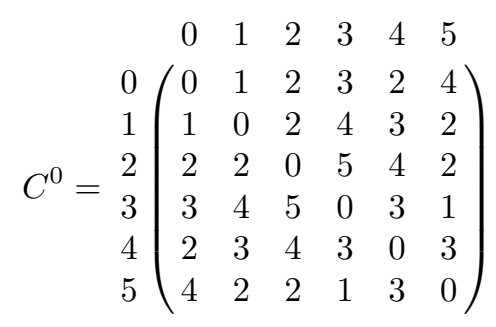

\subsection{Stage game}

In each stage game, all players choose actions simultaneously. Then the cost matrix associated with the graph $G\left(N^{\prime}, E\right)$ is defined.

Definition 4. A strategy of player $i$ is denoted by a vector

$$
x_{i}=\left(x_{i, 1}, \ldots, x_{i, i-1}, x_{i, i+1}, \ldots, x_{i, n}\right)
$$

where $x_{i, j}$ is a strategy of player $i$ against $j, \forall i \neq j \in N . X_{i, j}$ denotes the set of all strategies of player $i$ against $j, \forall x_{i, j} \in X_{i, j}$.

The cost of edge $(i, j)$ is defined as $c_{i j}=c_{j i}=f_{c}\left(x_{i, j}, x_{j, i}\right)$, where the cost function $f_{c}$ is a mapping from the set of strategies of players $i, j$ to the set of positive real numbers. So the cost of edge $(i, j)$ is determined by the player $i$ and $j, \forall i \neq j \in N$.

The cost of the edge between source $\{0\}$ and player $i \in N$ is equal to the cost of the edge in the initial cost matrix. i.e

$$
c_{i 0}=c_{0 i}=c_{i 0}^{0}=c_{0 i}^{0}>0
$$

For strategy profile $x=\left(x_{1}, x_{2}, \ldots, x_{n}\right)$, the cost matrix associated with $G\left(N^{\prime}, C_{x}\right)$ is denoted by $C_{x}=\left(c_{i j}\right)_{(n+1) \times(n+1)}$.

Definition 5. (Bird, 1976), the minimum cost spanning tree over $N^{\prime}$ is defined as follows

$$
T\left(N^{\prime}, C_{x}\right)=\arg \min _{G\left(N^{\prime}, E\right) \in \mathcal{G}_{N^{\prime}}} \sum_{(i, j) \in G\left(N^{\prime}, E\right)} c_{i j}
$$

where $C_{x}=\left(c_{i j}\right)_{(n+1) \times(n+1)}$ is the cost matrix defined by the strategy profile $x=$ $\left(x_{1}, x_{2}, \ldots, x_{n}\right), \forall i \neq j \in N$. 
Definition 6. The total cost of edges in the minimum cost spanning tree $T\left(N^{\prime}, C_{x}\right)$ is defined as

$$
C\left[T\left(N^{\prime}, C_{x}\right)\right]=\sum_{(i, j) \in T\left(N^{\prime}, C_{x}\right)} c_{i j}, \forall i \neq j \in N
$$

where $C_{x}=\left(c_{i j}\right)_{(n+1) \times(n+1)}$ is the cost matrix defined by strategy profile $x=$ $\left(x_{1}, x_{2}, \ldots, x_{n}\right)$.

Example 3. $N=\{1,2,3\}, N^{\prime}=N \cup\{0\}$. The sets of the strategies of the players are shown in Tab.1.

Table 1. The sets of the strategies

\begin{tabular}{|c|c|c|c|c|c|c|c|}
\hline \multirow[b]{2}{*}{$c_{12}$} & & $X_{2,1}$ & \multirow[b]{2}{*}{$c_{13}$} & $X_{3,1}$ & \multirow[b]{2}{*}{$c_{23}$} & \multicolumn{2}{|c|}{$X_{3,2}$} \\
\hline & & \begin{tabular}{l|l}
3 & 5
\end{tabular} & & \begin{tabular}{|l|l|}
2 & 5 \\
\end{tabular} & & 3 & 4 \\
\hline$X_{1}$, & 1 & \begin{tabular}{|l|l}
3 & 5 \\
\end{tabular} & $X_{1}$ & \begin{tabular}{|l|l|l|}
3 & 15 \\
\end{tabular} & $X_{2}$ & \begin{tabular}{|l|l}
2 & 6 \\
\end{tabular} & 8 \\
\hline & 2 & \begin{tabular}{|l|l|}
6 & 10
\end{tabular} & & \begin{tabular}{|l|l|}
4 & 8 \\
\end{tabular} & & \begin{tabular}{|l|l|}
4 & 12 \\
\end{tabular} & 16 \\
\hline
\end{tabular}

Assume that $f_{c}=x_{i, j} \times x_{j, i}, x_{i, j} \in X_{i, j}, x_{j, i} \in X_{j, i}, i \neq j \in N$. If player 1 chooses $x_{1,2}=1, x_{1,3}=3$, player 2 chooses $x_{2,1}=3, x_{2,3}=2$, and player 3 chooses strategy $x_{3,1}=2, x_{3,2}=3$. The cost of edge $(1,2)$ is $c_{12}=c_{21}=f_{c}\left(x_{1,2}, x_{2,1}\right)=x_{1,2} x_{2,1}=3$. Similarly, the cost of edge $(1,3)$ is $c_{13}=c_{31}=6$, and the cost of edge $(2,3)$ is $c_{23}=c_{32}=6$.

Thus, the cost matrix determined by the strategy profile $x=\left(x_{1}, x_{2}, \ldots, x_{3}\right)$ is

$$
C_{x}=\begin{aligned}
& 0 \\
& 1 \\
& 2 \\
& 3
\end{aligned}\left(\begin{array}{cccc}
0 & 1 & 2 & 3 \\
20 & 0 & 15 & 30 \\
15 & 3 & 0 & 6 \\
30 & 6 & 6 & 0
\end{array}\right)
$$

As shown in Fig.5, on the left side it is an entire graph $G\left(N^{\prime}, C_{x}\right)$ with cost. On the right side it is a minimum cost spanning tree $T\left(N^{\prime}, C_{x}\right)$ on the graph $G\left(N^{\prime}, C_{x}\right)$.
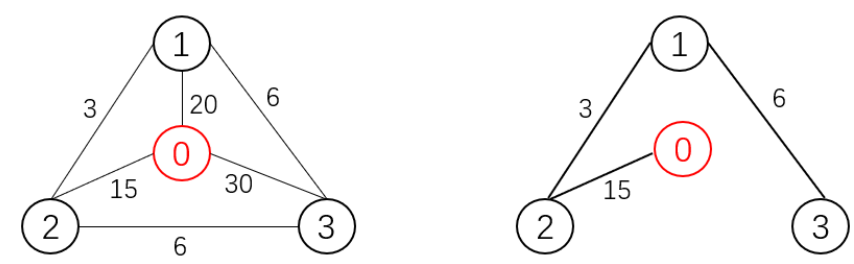

Fig. 5. $G\left(N^{\prime}, C_{x}\right)$ and $T\left(N^{\prime}, C_{x}\right)$

Thus, the total cost of edges on the minimum cost spanning tree $T\left(N^{\prime}, C_{x}\right)$ is $C\left(T\left(N^{\prime}, C_{x}\right)\right)=24$, the strategies profiles of player 1,2 and 3 are $x_{1}=(1,3), x_{2}=$ $(3,2), x_{3}=(2,3)$.

Consider two types of games with spanning trees on subgraph: "optimistic" game with spanning tree (Bergantiños et al., 2007b) and "pessimistic" game with spanning tree. 


\section{3. $\quad$ "pessimistic" game with spanning tree on subgraph}

The "pessimistic" game with spanning tree on subgraph involves the players in coalition $S \varsubsetneqq N$ connected with the source $\{0\}$ without any help from players outside of the coalition $S$.

Definition 7. (Bird, 1976) The minimum cost spanning tree on coalition $S \varsubsetneqq N$ in the "pessimistic" game with spanning tree is defined as

$$
T\left(S, C_{x_{S}}\right)=\arg \min _{G \in \mathcal{G}_{S^{\prime}}} \sum_{(i, j) \in G\left(S^{\prime}, E\right)} c_{i j}
$$

where $C_{x_{S}}$ is the cost matrix defined by strategy profile $x_{S}$.

Definition 8. The total cost of edges on the minimum cost spanning tree $T\left(S, C_{x}\right)$ is

$$
C\left[T\left(S, C_{x_{S}}\right)\right]=\sum_{(i, j) \in T\left(S, C_{x_{S}}\right)} c_{i j}
$$

where $C_{x_{S}}$ is the cost matrix defined by strategy profile $x_{S}$.

\section{4. "optimistic" game with spanning tree on subgraph}

In contrast to the above, in the optimistic game, if the coalition $S \varsubsetneqq N$ needs the help of the coalition $N \backslash S$, the total cost of the coalition $S$ consists of two parts, one is the total cost of spanning subtree of coalition $S$, and the other is the cost of connecting the coalition $S$ to the coalition $N \backslash S$. It means that using the connection to the source within coalition $N \backslash S$ is cost free for coalition $S$. In this research, if the coalition $N \backslash S$ supports the coalition S, the costs of the edges that will be provided between them are equal to the costs of the edges in the initial cost matrix.

Definition 9. The minimum cost spanning tree for coalition $S$ in the "optimistic" game is defined as follow

$$
\begin{gathered}
T^{+}\left(S, N^{\prime} \backslash S, C_{x_{S}}\right)=\arg \sum_{G(S, E) \in \mathcal{G}_{S}, G\left(N^{\prime}, E\right) \in \mathcal{G}_{N^{\prime}}}\left\{\sum_{(i, j) \in G(S, E)} c_{i j}\right. \\
\left.+\sum_{\left(o, o^{\prime}\right) \in G\left(N^{\prime}, E\right), o \in S, o^{\prime} \in N^{\prime} \backslash S} c_{o o^{\prime}}^{0}\right\}
\end{gathered}
$$

where $C_{x_{S}}$ is the cost matrix defined by strategy profile $x_{S}$.

Definition 10. The total cost of edges in the minimum cost spanning tree $T^{+}\left(S, N^{\prime} \backslash S, C_{x_{S}}\right)$ is

$$
C\left[T^{+}\left(S, N^{\prime} \backslash S, C_{x_{S}}\right)\right]=\sum_{(i, j) \in T^{+}\left(S, N^{\prime} \backslash S, C_{x_{S}}\right)} c_{i j}
$$

where $C_{x_{S}}$ is the cost matrix defined by strategy profile $x_{S}$.

Example 4. $N=\{1,2,3\}, N^{\prime}=N \cup\{0\}, S=\{2,3\}, f_{c}=x_{i, j} \times x_{j, i}, x_{i, j} \in$ $X_{i, j}, x_{j, i} \in X_{j, i}, i \neq j \in N$. As shown in Fig.6, player 2 and player 3 choose their actions $x_{2,3}=2, x_{3,2}=5$, thus the cost of edge $(2,3)$ is equal to 10 . The total 

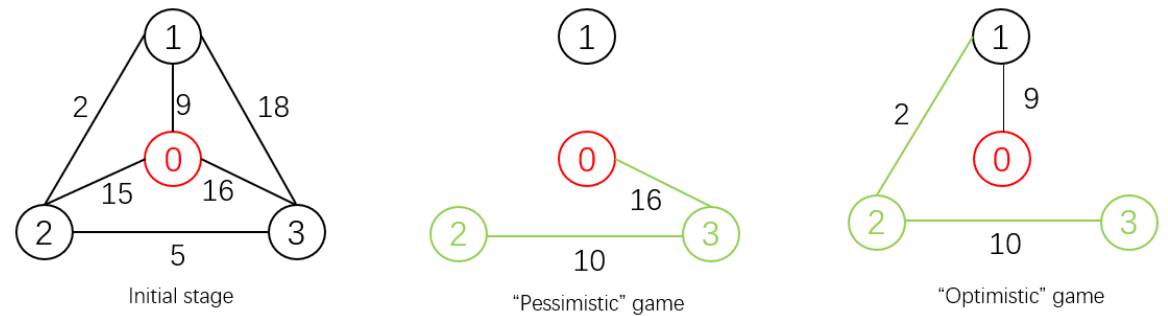

Fig. 6. "pessimistic" game and "optimistic" game on subgraph

cost of minimum cost spanning tree $T\left(S, C_{x_{S}}\right)$ is

$$
C\left[T\left(S, C_{x_{S}}\right)\right]=26
$$

and the total cost of minimum cost spanning tree $T^{+}\left(S, N^{\prime} \backslash S, C_{x_{S}}\right)$ is

$$
C\left[T^{+}\left(S, N^{\prime} \backslash S, C_{x_{S}}\right)\right]=12
$$

\subsection{Two-stage game with spanning tree}

Let $P_{m 0}$ denote the path from the source to $m$ on $G\left(N^{\prime}, E\right) . P(m)$ - an immediate predecessor of $m$ in the minimum cost spanning tree $T\left(N^{\prime}, C_{x}\right)$, if $P(m) \in P_{m 0}$ and $(P(m), m) \in T\left(N^{\prime}, C_{x}\right)$.

Definition 11. The probability of player $m$ leaving the game is defined as follows:

$$
p=\frac{\sum_{(i, j) \in B(m)} c_{i j}}{C\left[T\left(N^{\prime}, C_{x}\right)\right]}
$$

where $B(m)$ is the set of all edges of the subtree with root $m$.

The first stage: All players choose their strategies profiles simultaneously:

$$
\begin{gathered}
x^{1}=\left(x_{1}^{1}, x_{2}^{1}, \ldots, x_{n}^{1}\right) \\
x_{i}^{1}=\left(x_{i, 1}^{1}, x_{i, 2}^{1}, \ldots, x_{i, i-1}^{1}, x_{i, i+1}^{1}, \ldots, x_{i, n}^{1}\right)
\end{gathered}
$$

where $x_{i, j}^{1}$ is an action of player $i$ against $j, \forall i \neq j \in N$.

The second stage: Since the player $m$ may leave the game, there are two types of stage game.

If $m$ does not exit the game, all players choose their strategy profiles at the same time, just as they behaved in the first stage:

$$
\begin{gathered}
x^{2}=\left(x_{1}^{2}, x_{2}^{2}, \ldots, x_{n}^{2}\right) \\
x_{i}^{2}=\left(x_{i, 1}^{2}, x_{i, 2}^{2}, \ldots, x_{i, i-1}^{2}, x_{i, i+1}^{2}, \ldots, x_{i, n}^{2}\right)
\end{gathered}
$$

where $x_{i, j}^{2}$ is an action of player $i$ against $j$ and $x_{i, j}^{2} \in X_{i, j}^{2}, \forall i \neq j \in N$.

If the player $m$ leaves the game. The players choose their strategies profiles:

$$
\begin{gathered}
x^{2} \backslash\{m\}=\left(x_{1}^{2}, x_{2}^{2}, \ldots, x_{m-1}^{2}, x_{m+1}^{2}, \ldots, x_{n}^{2}\right) \\
x_{i}^{2}=\left(x_{i, 1}^{2}, x_{i, 2}^{2}, \ldots, x_{i, i-1}^{2}, x_{i, i+1}^{2}, \ldots, x_{i, n}^{2}\right)
\end{gathered}
$$

where $x_{i, j}^{2}$ is an action of player $i$ against $j$ and $x_{i, j}^{2} \in X_{i, j}^{2}, \forall i \neq j \in N \backslash\{m\}$. 


\section{Cooperative game}

Now we define the characteristic function for the grand coalition $N$ in the game. Let $x(\cdot)$ denote the strategy of players in the game.

$$
\begin{aligned}
V^{1}\left(N^{\prime}\right) & =\min _{x(\cdot)}\left\{C\left[T\left(N^{\prime}, C_{x^{1}}\right)\right]+\left[p C\left[T\left(N^{\prime} \backslash\{m\}, C_{x^{2} \backslash\{m\}}\right)\right]+(1-p) C\left[T\left(N^{\prime}, C_{x^{2}}\right)\right]\right]\right\} \\
= & C\left[T\left(N^{\prime}, C_{\bar{x}^{1}}\right)\right]+\left[p C\left[T\left(N^{\prime} \backslash\{m\}, C_{\bar{x}^{2} \backslash\{m\}}\right)\right]+(1-p) C\left[T\left(N^{\prime}, C_{\bar{x}^{2}}\right)\right]\right]
\end{aligned}
$$

where $p=\frac{\sum_{(i, j) \in B(m)} c_{i j}}{C\left[T\left(N^{\prime}, C_{\bar{x}^{1}}\right)\right]}, \bar{x}_{i}(\cdot)$ is the cooperative strategy of player $i$, and the strategy profile $\bar{x}(\cdot)$ is called cooperative strategy profile.

Then define the characteristic function for the grand coalition $N$ in subgame.

If player $m$ leaves the game after the first stage,

$$
\begin{gathered}
V^{2}\left(N^{\prime} \backslash\{m\}\right)=\min _{x^{2}(\cdot)} C\left[T\left(N^{\prime} \backslash\{m\}, C_{x^{2} \backslash\{m\}}\right)\right] \\
=C\left[T\left(N^{\prime} \backslash\{m\}, C_{\bar{x}^{2}}\right)\right]
\end{gathered}
$$

where $\bar{x}_{i}^{2}(\cdot), i \in N \backslash\{m\}$ are the cooperative strategies, and the cooperative strategy profile is $\bar{x}^{2}(\cdot)$.

If player $m$ does not leave the game,

$$
V^{2}\left(N^{\prime}\right)=\min _{x^{2}(\cdot)} C\left[T\left(N^{\prime}, C_{x^{2}}\right)\right]=C\left[T\left(N^{\prime}, C_{\bar{x}^{2}}\right)\right]
$$

where $\bar{x}_{i}^{2}(\cdot), i \in N$ are cooperative strategies, and the cooperative strategy profile is $\bar{x}^{2}(\cdot)$.

\subsection{The characteristic function in "pessimistic" game with spanning tree}

By the Bird(Bird, 1976) method, the characteristic function for the coalition $S \varsubsetneqq N$ is defined.

If $m \in S$,

$$
\begin{aligned}
V^{1}\left(S^{\prime}\right) & =\min _{x_{S}(\cdot)}\left\{C\left[T\left(S, C_{x_{S}^{1}}^{S}\right)\right]+\left[p C\left[T\left(S \backslash\{m\}, C_{x_{S}^{2} \backslash\{m\}}^{S \backslash\{m\}}\right)\right]+(1-p) C\left[T\left(S, C_{x_{S}^{2}}^{S}\right)\right]\right]\right\} \\
& =C\left[T\left(S, C_{\bar{x}_{S}^{1}}^{S}\right)\right]+\left[p C\left[T\left(S \backslash\{m\}, C_{\bar{x}_{S}^{2} \backslash\{m\}}^{S \backslash\{m\}}\right)\right]+(1-p) C\left[T\left(S, C_{\bar{x}_{S}^{2}}^{S}\right)\right]\right]
\end{aligned}
$$

where $p=\frac{\sum_{(i, j) \in B(m)} c_{i j}}{C\left[T\left(S, C_{x_{S}^{1}}^{S}\right)\right]}, C^{S}$ and $C^{S \backslash\{m\}}$ is the cost matrix restricted to $S$ and $S \backslash\{m\}$.

If $m \notin S$,

$$
V^{1}\left(S^{\prime}\right)=\min _{x_{S}(\cdot)}\left[C\left[T\left(S, C_{x_{S}^{1}}^{S}\right)\right]+C\left[T\left(S, C_{x_{S}^{2}}^{S}\right)\right]\right]=C\left[T\left(S, C_{\bar{x}_{S}^{1}}^{S}\right)\right]+C\left[T\left(S, C_{\bar{x}_{S}^{2}}^{S}\right)\right]
$$

where $C^{S}$ and $C^{S \backslash\{m\}}$ is the cost matrix restricted to $S$ and $S \backslash\{m\}$.

Since player $m$ will never leave the game, the value of the characteristic function for the coalition $S$ is equal to the total cost of edges in the minimum costs spanning tree over $S^{\prime}$ :

$$
V^{2}\left(S^{\prime}\right)=\min _{x_{S}(\cdot)} C\left[T\left(S^{\prime}, C_{x_{S}^{2}}^{S^{\prime}}\right)\right]=C\left[T\left(S^{\prime}, C_{\bar{x}_{S}^{2}}^{S^{\prime}}\right)\right]
$$

where $S \subset N, S^{\prime}=S \cup\{0\}, C^{S^{\prime}}$ is the cost matrix restricted to $S^{\prime}$. 


\subsection{The characteristic function in "optimistic" game with spanning tree}

The characteristic function for coalition $S$ is defined as follows.

If $m \in S, S \varsubsetneqq N$, and $S^{\prime}=S \cup\{0\}$

$$
\begin{gathered}
V^{1+}\left(S^{\prime}\right)=\min _{x_{S}(\cdot)}\left\{C\left[T^{+}\left(S, N^{\prime} \backslash S, C_{x_{S}^{1}}^{S}\right)\right]+\left[p C\left[T^{+}\left(S \backslash\{m\}, N^{\prime} \backslash S \backslash\{m\}, C_{x_{S}^{2} \backslash\{m\}}^{S \backslash m\}}\right)\right]\right.\right. \\
\left.\left.+(1-p) C\left(T^{+}\left(N^{\prime}, C_{x_{S}^{2}}^{S}\right)\right)\right]\right\} \\
=C\left[T^{+}\left(S, N^{\prime} \backslash S, C_{\bar{x}_{S}^{1}}^{S}\right)\right]+\left[p C\left[T^{+}\left(S \backslash\{m\}, N^{\prime} \backslash S \backslash\{m\}, C_{\bar{x}_{S}^{2} \backslash\{m\}}^{S \backslash\{m\}}\right)\right]\right. \\
\left.+(1-p) C\left[T^{+}\left(S, N^{\prime} \backslash S, C_{\bar{x}_{S}^{2}}^{S}\right)\right]\right]
\end{gathered}
$$

where $p=\frac{\sum_{(i, j) \in B(m)} c_{i j}}{C\left[T^{+}\left(S, N^{\prime} \backslash S, C_{x_{S}^{1}}^{S}\right)\right]}, C^{S^{\prime}}$ and $C^{S^{\prime} \backslash\{m\}}$ is the cost matrix restricted to $S^{\prime}$ and $S^{\prime} \backslash\{m\}$.

If $m \notin S, S \varsubsetneqq N$, and $S^{\prime}=S \cup\{0\}$

$$
\begin{aligned}
V^{1+}\left(S^{\prime}\right) & =\min _{x_{S}(\cdot)}\left[C\left[T^{+}\left(S, N^{\prime} \backslash S, C_{x_{S}^{1}}^{S}\right)\right]+C\left[T^{+}\left(S, N^{\prime} \backslash S, C_{x_{S}^{2}}^{S}\right)\right]\right] \\
= & C\left[T^{+}\left(S, N^{\prime} \backslash S, C_{\bar{x}_{S}^{1}}^{S}\right)\right]+C\left[T^{+}\left(S, N^{\prime} \backslash S, C_{\bar{x}_{S}^{2}}^{S}\right)\right]
\end{aligned}
$$

where $C^{S}$ is the cost matrix restricted to $S, C^{S^{\prime}}$ is the cost matrix restricted to $S^{\prime}$.

The characteristic function for the coalition $S$ in subgame:

$$
V^{2+}\left(S^{\prime}\right)=\min _{x_{S}(\cdot)} C\left[T^{+}\left(S, N^{\prime} \backslash S, C_{x_{S}^{2}}^{S}\right)\right]=C\left[T^{+}\left(S, N^{\prime} \backslash S, C_{\bar{x}_{S}^{2}}^{S}\right)\right]
$$

where $C^{S^{\prime}}$ is the cost matrix restricted to $S^{\prime}$.

\section{The Shapley value in the two stage "optimistic" game with spanning tree.}

On the basis of the above discussion, we can define Shapley values in the two stage "optimistic" game with spanning tree.

$$
S h_{i}^{1+}\left(N^{\prime}, C\right)=\frac{1}{n !} \sum_{\pi \in \Pi}\left[V^{1+}\left(S_{\pi(i)}^{\prime} \cup\{i\}\right)-V^{1+}\left(S_{\pi(i)}^{\prime}\right)\right]
$$

where $\Pi$ denotes the set of all permutations on $N$, and $S_{\pi(k)}=\{i \mid \pi(i)<\pi(k)\}$.

In the similar way, the Shapley value in subgame is defined as follows.

If player $m$ leaves the game after the first stage:

$$
S h_{i}^{2+}\left(N^{\prime} \backslash\{m\}, C\right)=\frac{1}{(n-1) !} \sum_{\pi^{\prime} \in \Pi^{\prime}}\left[V^{2+}\left(S_{\pi^{\prime}(i)}^{\prime} \cup\{i\}\right)-V^{2+}\left(S_{\pi^{\prime}(i)}^{\prime}\right)\right]
$$

where $\Pi^{\prime}$ denotes the set of all permutations on $N \backslash\{m\}$, and $S_{\pi^{\prime}(k)}=\left\{i \mid \pi^{\prime}(i)<\right.$ $\left.\pi^{\prime}(k)\right\}$.

If player $m$ doesn't leave the game after the first stage:

$$
S h_{i}^{2+}\left(N^{\prime}, C\right)=\frac{1}{n !} \sum_{\pi \in \Pi}\left[V^{2+}\left(S_{\pi(i)}^{\prime} \cup\{i\}\right)-V^{2+}\left(S_{\pi(i)}^{\prime}\right)\right]
$$

where $\Pi$ denotes the set of all permutations on $N$, and $S_{\pi(k)}=\{i \mid \pi(i)<\pi(k)\}$. 


\section{Results}

\section{Theorem 1.}

$$
C\left[T^{+}\left(S, N^{\prime} \backslash S, C_{x_{S}}\right)\right] \leq C\left[T\left(S^{\prime}, N \backslash S^{\prime}, C_{x_{S}}\right)\right], S \varsubsetneqq N
$$

where $C_{x_{S}}$ is the cost matrix defined by strategy profile $x_{S}$.

Proof. By definition 9, there are only two possible forms of the minimum cost spanning tree $T^{+}\left(S, N^{\prime} \backslash S, C_{x_{S}}\right)$. The first case is the coalition $S$ connected directly to the source; the second case is connected via one or several vertexes in the coalition $N \backslash S$.

Case 1: if the coalition $S$ connected directly to the source $\{0\}$, the minimum cost spanning tree $T^{+}\left(S, N^{\prime} \backslash S, C_{x_{S}}\right)$ is over $S^{\prime}=S \cup\{0\}$. On the graph $G\left(S^{\prime}, E\right)$ with cost matrix $C_{x_{S}}$, the "optimistic" minimum cost spanning tree is the same to "pessimistic" minimum cost spanning tree, $T^{+}\left(S, N^{\prime} \backslash S, C_{x_{S}}\right)=T\left(S, N^{\prime} \backslash S, C_{x_{S}}\right)$. i.e.

$$
C\left[T^{+}\left(S, N^{\prime} \backslash S, C_{x_{S}}\right)\right]=C\left[T\left(S^{\prime}, N \backslash S^{\prime}, C_{x_{S}}\right)\right]
$$

Case 2: if the coalition $S$ connected with the source $\{0\}$ via one or several vertexes in the coalition $N \backslash S$ over $N^{\prime}$, consider using the converse method, such that the total cost of $T^{+}\left(S, N^{\prime} \backslash S, C_{x_{S}}\right)$ is larger than the total cost of $T\left(S, N^{\prime} \backslash S, C_{x_{S}}\right)$. i.e.

$$
C\left[T^{+}\left(S, N^{\prime} \backslash S, C_{x_{S}}\right)\right]>C\left[T\left(S^{\prime}, N \backslash S^{\prime}, C_{x_{S}}\right)\right], S \varsubsetneqq N
$$

Then the coalition $S$ can achieve the case where it is connected to source $\{0\}$ and reduces the total cost by connecting directly to source $\{0\}$ and removing the edges associated with the vortexes in $N \backslash S$, i.e. such case $\left(C\left[T^{+}\left(S, N^{\prime} \backslash S, C_{x_{S}}\right)\right]>\right.$ $\left.C\left[T\left(S^{\prime}, N \backslash S^{\prime}, C_{x_{S}}\right)\right], S \varsubsetneqq N\right)$ does not exist.

The theorem is proven.

\section{Theorem 2.}

$$
C\left[T^{+}\left(S, N^{\prime} \backslash S, C_{x_{S}}\right)\right] \leqslant C\left[T^{+}\left(S, N^{\prime} \backslash\{m\} \backslash S, C_{x_{S}}\right)\right], S \varsubsetneqq N
$$

where $C_{x_{S}}$ is the cost matrix defined by strategy profile $x_{S}$.

Proof. Two cases are considered in the minimum cost spanning tree $T^{+}\left(S, N^{\prime} \backslash\right.$ $\left.S, C_{x_{S}}\right)$ obtained on the graph $G\left(S^{\prime}, E\right)$ with cost matrix $C_{x_{S}}$ : the first case, in which the help of the player $m$ is needed, i.e. the minimum spanning tree $T^{+}\left(S, N^{\prime} \backslash S, C_{x_{S}}\right)$ contains $m$; and the second case, in which the help of the player $m$ is not needed.

Case 1: if the coalition $S$ connected to the source $\{0\}$ with the help of the player $m$, the minimum cost spanning tree $T^{+}\left(S, N^{\prime} \backslash S, C_{x_{S}}\right)$ can be represented as

$$
\begin{gathered}
T^{+}\left(S, N^{\prime} \backslash S, C_{x_{S}}\right)=\arg \min _{G(S, E) \in \mathcal{G}_{S}, G\left(N^{\prime}, E\right) \in \mathcal{G}_{N^{\prime}}}\left\{\sum_{(i, j) \in G(S, E)} c_{i j}\right. \\
\left.+\sum_{\left(o, o^{\prime}\right) \in G\left(N^{\prime} \backslash\{m\}, E\right), o \in S, o^{\prime} \in N^{\prime} \backslash S \backslash\{m\}} c_{o o^{\prime}}^{0}+c_{o m^{\prime}}^{0}\right\}
\end{gathered}
$$

where $C_{x_{S}}$ is the cost matrix defined by strategy profile $x_{S}$.

After the player $m$ leaves the game, there will be three possible changes to the new minimum cost spanning tree $T^{+}\left(S, N^{\prime} \backslash\{m\} \backslash S, C_{x_{S}}\right)$. 
- If the total cost of the minimum cost spanning tree $T^{+}\left(S, N^{\prime} \backslash\{m\} \backslash S, C_{x_{S}}\right)$ that does not contain the player $m$ is equal to the total cost of the previous minimum cost spanning tree $T^{+}\left(S, N^{\prime} \backslash S, C_{x_{S}}\right)$, i.e.

$$
C\left[T^{+}\left(S, N^{\prime} \backslash S, C_{x_{S}}\right)\right]=C\left[T^{+}\left(S, N^{\prime} \backslash\{m\} \backslash S, C_{x_{S}}\right)\right], S \varsubsetneqq N
$$

- If the total cost of the minimum cost spanning tree $T^{+}\left(S, N^{\prime} \backslash\{m\} \backslash S, C_{x_{S}}\right)$ that does not contain the player $m$ is larger than to the total cost of the previous minimum cost spanning tree $T^{+}\left(S, N^{\prime} \backslash S, C_{x_{S}}\right)$, i.e.

$$
C\left[T^{+}\left(S, N^{\prime} \backslash S, C_{x_{S}}\right)\right]<C\left[T^{+}\left(S, N^{\prime} \backslash\{m\} \backslash S, C_{x_{S}}\right)\right], S \varsubsetneqq N
$$

- If the total cost of the minimum cost spanning tree $T^{+}\left(S, N^{\prime} \backslash\{m\} \backslash S, C_{x_{S}}\right)$ that does not contain the player $m$ is less than to the total cost of the previous minimum cost spanning tree $T^{+}\left(S, N^{\prime} \backslash S, C_{x_{S}}\right)$, i.e.

$$
C\left[T^{+}\left(S, N^{\prime} \backslash S, C_{x_{S}}\right)\right]<C\left[T^{+}\left(S, N^{\prime} \backslash\{m\} \backslash S, C_{x_{S}}\right)\right], S \varsubsetneqq N
$$

By Definition 9, it means that the minimum spanning tree game $T^{+}\left(S, N^{\prime} \backslash\right.$ $S, C_{x_{S}}$ ) for coalition $S$ is not the minimum total cost, since a much less total cost can be achieved by excluding the edges associated with $m$. This contradicts the statement of the theorem. Therefore this case does not exist.

Case 2: Assume that the players in the coalition $S$ is not connected to the source $\{0\}$ with the help of the player $m$,

$$
C\left[T^{+}\left(S, N^{\prime} \backslash S, C_{x_{S}}\right)\right]=C\left[T^{+}\left(S, N^{\prime} \backslash\{m\} \backslash S, C_{x_{S}}\right)\right]
$$

The theorem is proven.

\section{Example}

The set of players is $N=1,2,3$, the source is $\{0\}, N^{\prime}=N \cup\{0\}$. Assume that player 3 may leave the game after stage 1. As shown in Fig.7, there are initial costs of the edges between each player.

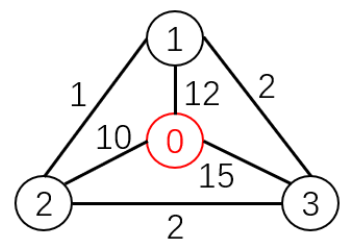

Fig. 7. The graph $G(N, E)$ with initial costs of the edges

The initial cost matrix $C^{0}$ is

$$
C^{0}=\begin{aligned}
& 0 \\
& 1 \\
& 2 \\
& 3
\end{aligned}\left(\begin{array}{cccc}
0 & 1 & 2 & 3 \\
12 & 0 & 1 & 15 \\
10 & 1 & 0 & 2 \\
15 & 2 & 2 & 0
\end{array}\right)
$$


Table 2. Strategy set

\begin{tabular}{|c|c|c|c|c|c|c|c|c|c|c|c|}
\hline \multirow{2}{*}{$c_{12}$} & & $X_{2}$ & & & \multicolumn{3}{|c|}{$X_{3,1}$} & & \multicolumn{2}{|r|}{$X_{3,2}$} \\
\hline & & \begin{tabular}{l|l}
2 & 4 \\
\end{tabular} & 6 & & & 2 & 3 & 7 & & 1 & \begin{tabular}{|l|l|}
4 & 6 \\
\end{tabular} \\
\hline & & \begin{tabular}{l|l}
2 & 4 \\
\end{tabular} & 6 & & 2 & 4 & 6 & 14 & & & \begin{tabular}{|l|l|}
12 & 18 \\
\end{tabular} \\
\hline$r_{1,2}$ & & \begin{tabular}{l|l}
6 & 12
\end{tabular} & \begin{tabular}{l|l}
2 & 18
\end{tabular} & $X_{1,3}$ & 3 & 6 & \begin{tabular}{|l|}
9 \\
\end{tabular} & 21 & $X _ { 2 , 3 } \longdiv { 6 }$ & & \begin{tabular}{|l|l|}
24 & 36 \\
\end{tabular} \\
\hline & & \begin{tabular}{l|l|}
10 & 20 \\
\end{tabular} & 030 & & 5 & 10 & & 35 & 7 & \begin{tabular}{l|l|l|}
7 & 7
\end{tabular} & \begin{tabular}{|l|l|}
28 & 42 \\
\end{tabular} \\
\hline
\end{tabular}

The strategy set for each player is shown in Tab. 2 .

Assume the function $f_{c}=x_{i, j} \times x_{j, i}$. For example, if player 1 choose action $x_{1,2}=3$ and player 2 choose action $x_{2,1}=4$. The cost of edge $(1,2)$ will be $c_{12}=f_{c}\left(x_{1,2}, x_{2,1}\right)=x_{1,2} x_{2,1}=12$.

The cooperative strategies in the game are:

$$
\begin{aligned}
& \bar{x}_{1}=\left(\bar{x}_{1}^{1}, \bar{x}_{1}^{2}\right)=((1,2),(1,2)) \\
& \bar{x}_{2}=\left(\bar{x}_{2}^{1}, \bar{x}_{2}^{2}\right)=((2,3),(2,3)) \\
& \bar{x}_{3}=\left(\bar{x}_{3}^{1}, \bar{x}_{3}^{2}\right)=((2,1),(2,1))
\end{aligned}
$$

The probability of player 3 leaving the game is $p=0.467$ and $V^{1}(N)=28.599$.

The characteristic function for each coalition and the Shapley value in two-stage "optimistic" game with spanning tree:

$$
\begin{gathered}
V^{1+}(\{1\})=4, V^{1+}(\{2\})=4, V^{1+}(\{3\})=4.599 \\
V^{1+}(\{1,2\})=10, V^{1+}(\{1,3\})=10.132, V^{1+}(\{2,3\})=8.599 \\
S h_{1}^{1+}\left(N^{\prime}, C\right)=9.922, S h_{2}^{1+}\left(N^{\prime}, C\right)=9.156, S h_{3}^{1+}\left(N^{\prime}, C\right)=9.442
\end{gathered}
$$

Similarly, the characteristic function for each coalition and Shapley value in subgame:

If player $m$ doesn't leave the game

$$
\begin{gathered}
V^{2}\left(N^{\prime}\right)=15, V^{2+}(\{1\})=2, V^{2+}(\{2\})=2 \\
V^{2+}(\{3\})=3, V^{2+}(\{1,2\})=4, V^{2+}(\{1,3\})=5, V^{2+}(\{2,3\})=4 \\
S h_{1}^{2+}\left(N^{\prime}, C\right)=5, S h_{2}^{2+}\left(N^{\prime}, C\right)=4.5, S h_{3}^{2+}\left(N^{\prime}, C\right)=5.5
\end{gathered}
$$

If player $m$ leaves the game

$$
\begin{gathered}
V^{2}\left(N^{\prime}\right)=12, V^{2+}(\{1\})=2, V^{2+}(\{2\})=2 \\
V^{2+}(\{3\})=0, V^{2+}(\{1,2\})=12, V^{2+}(\{1,3\})=0, V^{2+}(\{2,3\})=0 \\
S h_{1}^{2+}\left(N^{\prime}, C\right)=6, S h_{2}^{2+}\left(N^{\prime}, C\right)=6, S h_{3}^{2+}\left(N^{\prime}, C\right)=0
\end{gathered}
$$

\section{Conclusion}

This paper considers the Shapley value in a two-stage "optimistic" game with a spanning tree. It assumes that players in coalition $S$ may indirectly connect to the source with the help of players, not in the coalition $S$. The definition of Shapley value in the "optimistic" game with spanning tree is given. Several theorems and examples are provided. 
New Characteristic Function for Two Stage Games with Spanning Tree

\section{References}

Bird, C.G. (1976). On cost allocation for a spanning tree: a game theoretic approach. Networks., 6(4), 335-350.

Bergantiños, G. and Vidal-Puga J J. (2007). The optimistic TU game in minimum cost spanning tree problems. International Journal of Game Theory., 36(2), 223-239.

Yin, L. (2016). The dynamic Shapley value in the game with spanning tree. In: 2016 International Conference Stability and Oscillations of Nonlinear Control Systems (Pyatnitskiy's Conference)., pp. 1-4. IEEE.

Yin, L. (2017). Dynamic Shapley value in the game with spanning forest. In: 2017 Constructive Nonsmooth Analysis and Related Topics (dedicated to the memory of VF Demyanov)(CNSA)., pp. 1-4. IEEE.

Yin, L. (2017). Dynamic Shapley value for 2-stage cost sharing game with perishable products. In: 2017 29th Chinese Control and Decision Conference (CCDC)., pp. 3770-3774. IEEE.

Kar, A. (2002). Axiomatization of the Shapley value on minimum cost spanning tree games. Games and Economic Behavior., 38(2), 265-277.

Yin, L. (2020). The dynamic Nash bargaining solution for 2-stage cost sharing game. Contributions to Game Theory and Management., 13(0), 296-303.

Granot, D. and G. Huberman (1984). On the core and nucleolus of minimum cost spanning tree games. Mathematical Programming., 29, 323-347.

Feltkamp, V., S. Tijs, and S. Muto, (1994). On the irreducible core and the equal remaining obligations rule of minimum cost spanning extension problems. Center for Economic Research Discussion Paper 94106., Tilburg University.

Bergantiños, G. and Vidal-Puga J J. (2007). A fair rule in minimum cost spanning tree problems. Journal of Economic Theory., 137(1), 326-352.

Petrosyan, L. A., and Danilov, N. N. (1979). Stability of solutions in non-zero sum differential games with transferable payoffs. Viestnik of Leningrad Universtiy, 1, pp. 52-59. 